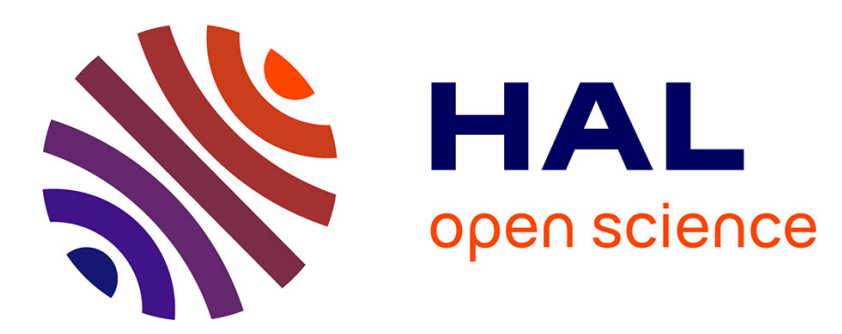

\title{
Bottom-up model for understanding the effects of wheat endosperm microstructure on its mechanical strength
}

Emna Chichti, Valerie Lullien-Pellerin, Matthieu George, Farhang Radjai, Rafik Affes, Jean-Yves Delenne

\section{- To cite this version:}

Emna Chichti, Valerie Lullien-Pellerin, Matthieu George, Farhang Radjai, Rafik Affes, et al.. Bottomup model for understanding the effects of wheat endosperm microstructure on its mechanical strength. International Journal of Food Engineering, 2016, 190, pp.40-47. 10.1016/j.jfoodeng.2016.06.009 hal01414119

\author{
HAL Id: hal-01414119 \\ https://hal.science/hal-01414119
}

Submitted on 15 Nov 2017

HAL is a multi-disciplinary open access archive for the deposit and dissemination of scientific research documents, whether they are published or not. The documents may come from teaching and research institutions in France or abroad, or from public or private research centers.
L'archive ouverte pluridisciplinaire $\mathbf{H A L}$, est destinée au dépôt et à la diffusion de documents scientifiques de niveau recherche, publiés ou non, émanant des établissements d'enseignement et de recherche français ou étrangers, des laboratoires publics ou privés. 


\title{
Bottom-up model for understanding the effects of wheat endosperm microstructure on its mechanical strength
}

\author{
Emna Chichti a, Valérie Lullien-Pellerin ${ }^{a}$, Matthieu George ${ }^{c}$, Farhang Radjai b, d, \\ Rafik Affès ${ }^{b}$, Jean-Yves Delenne ${ }^{a, *}$ \\ a UMR IATE, CIRAD, INRA, Montpellier SupAgro, Université de Montpellier, F-34060 Montpellier, France \\ ${ }^{\mathrm{b}}$ LMGC UMR 5508 CNRS-Université de Montpellier, 163 rue Auguste Broussonnet, 34090 Montpellier, France \\ ${ }^{c}$ Laboratoire Charles Coulomb, UMR 5221, CNRS-Université de Montpellier, 163 rue Auguste Broussonnet, 34090 Montpellier, France \\ d $\langle\text { MSE }\rangle^{2}$ UMI 3466 CNRS-MIT, DCEE, Massachusetts Institute of Technology, 77 Massachusetts Avenue, Cambridge, CA 02139, USA
}

\begin{abstract}
Wheat flours are essential ingredients of daily food products like bread, cookies or pastries. Their quality depends on the milling process and mechanical strength of wheat grains. Although it is well known that the strength and rupture of grains are strongly controlled by the endosperm microstructure, the respective roles of the starch and polymer volume fractions and their adhesion are not yet fully un-derstood. This typical biological microstructure can be modeled as a cemented granular material, where the two size populations of starch granules (large:A-type, small:B-type) are the particles, and the protein matrix, which partially fills the space between granules, plays the role of a cement. This structural model of wheat endosperm is used, together with mechanical characteristics of starch and proteins obtained by means of Atomic Force Microscopy (AFM) measurements, to simulate the mechanical behavior and breakage of wheat endosperm in milling process. We find that the porosity outweighs the effect of other parameters for the elastic modulus, which declines as a nearly linear function of porosity. We also show that the tensile strength is an increasing function of the amount and connectivity of starch granules with increasing concentration of stresses along chains of granules. This effect is more significant at low porosity where stress distribution is mainly controlled by the contact network between starch granules. This effect explains why the protein content is not fully correlated to vitreousness, and samples of similar protein content can be different in vitreosity. Finally, we find that the starch-granule adhesion strongly affects the tensile strength whereas the effect of starch volume fraction appears mainly at high interface adhesion, which is the case of hard type wheat grains.
\end{abstract}

\author{
Keywords: \\ Wheat endosperm \\ Hardness \\ Vitreousness \\ Cemented granular material \\ Failure \\ Numerical modeling
}

\section{Introduction}

Wheat flour characteristics such as particle size distribution and starch damage level are important factors for food product quality (Pasha et al., 2010). Such characteristics arise from the mechanical properties of the starchy endosperm, which is made of starch granules embedded in a protein matrix (Evers and Millar, 2002), and the milling process. The observed differences in flour properties are generally attributed to two main wheat grain characteristics: hardness and vitreousness (Haddad et al., 2001; Greffeuille et al., 2006, 2007). Wheat hardness represents the potential of

\footnotetext{
* Corresponding author.

E-mail address: jean-yves.delenne@supagro.inra.fr (J.-Y. Delenne).
}

producing fractions from a specific mechanical loading. It is usually estimated from a controlled grinding operation and a measure of particle size distribution (Williams and Sobering, 1986). Hence, from a mechanical viewpoint, wheat hardness reflects the fracture energy of wheat grains in response to mechanical loading during the grinding process (Wang and Jeronimidis, 2008).

Wheat hardness is a genetic-controlled property mainly linked to the Ha locus on the short arm of chromosome 5D (Turnbull and Rahman, 2002) and there is increasing evidence that it expresses itself through differences in starch-protein adhesion (Barlow et al., 1973; Greenwell and Schofield, 1986; Glenn and Johnston, 1992). Furthermore, these differences in adhesion depends on the nature and content of specific proteins, called puroindolines, present at the starch-granule interface (Turnbull and Rahman, 2002; Morris, 2002). The presence of the wild type version of puroindolines 
leads to a soft mechanical behavior due to a low adhesion between starch granules and the protein network, whereas mutation or deletion of one or both of the puroindolines genes results in a hard texture (Hogg et al., 2004; Giroux and Morris, 1998; Beecher et al., 2002).

Grain vitreousness is an optical property related to endosperm translucence and it characterizes the mealy and vitreous states of the starchy endosperm (Anjum and Walker, 1991; Weightmann et al., 2008). It is mainly controlled by the environmental conditions during growing and grain maturation (Parish and Halse, 1969; Oury et al., 2015; Lopez-Ahumada et al., 2010) which affect the grain density via the proportion of voids in the endosperm (Anjum and Walker, 1991; Dobraszczyk et al., 2002). If vitreousness was generally significantly correlated with the protein content (Weightmann et al., 2008; Lopez-Ahumada et al., 2010), this relationship remains under debate as different levels of vitreousness can be observed for grains displaying the same protein content (Greffeuille et al., 2006, 2007).

This means that the volume fraction of starch, which is the main constituent of wheat endosperm whose accumulation during grain development is also affected by growing conditions (Dai et al., 2009; Zhang et al., 2010; Hurkman and Wood, 2011; Ni et al., 2012), may well affect grain vitreousness. Therefore, starch particle content or its size distribution are expected to play a role in vitreousness and hence in the milling behavior of wheat grains. However, this factor has not yet been fully investigated on quantitative grounds.

In this paper, we analyze the role of starch and protein volume fractions with respect to the mechanical properties of wheat endosperm by means of numerical simulations. The mechanical model is a particulate system in which the starch granules are embedded in a porous protein matrix. Two different methods have been applied to simulate this model. In (Delenne et al., 2008), the authors used the Discrete Element Method (DEM) in which the protein matrix is introduced only through its cohesive action between starch granules (Cundall and Strack, 1979). This approach accounts for the effect of internal damage on the elastic and failure properties of endosperm. More recently, the Lattice Element Method (LEM) was used for a detailed parametric study of wheat endosperm (Topin et al., 2007; Affes et al., 2012). In this method, both starch granules and protein are represented as continuous material phases. The authors investigated the influence of particlematrix adhesion and protein content on strength and failure properties of the endosperm. Three distinct crack regimes were established, and the importance of starch-protein adhesion and protein content for crack propagation and fraction properties were quantitatively analyzed.

The LEM has the advantage of allowing for a realistic representation of the endosperm texture. But it needs as its input parameters the elastic constants of the starch granules and protein matrix, as well as the adhesion at the interfaces between starch granules and between the matrix and starch granules. In the previous studies (Topin et al., 2008), the mechanical properties of starch and protein phases were derived from the work of (Barlow et al., 1973; Glenn and Johnston, 1992), where micro-indentation tests were performed on wheat grain sections or individual polymers included in a resin. The resulting mechanical properties of starch and proteins appeared to be weakly contrasted and were thus considered to be identical. However, recent work of (Chichti et al., 2013) based on Atomic Force Microscopy (AFM) measurements, revealed that the mechanical properties of the phases differed from those used by (Topin et al., 2008).

In this paper, we use a $2 D$ Lattice Element Method together with our AFM measurements of phase properties, in order to analyze the effects of starch and protein volume fractions with respect to shear strength and effective elastic behavior of wheat endosperm. After a brief description of the numerical model and methodology used to prepare the samples, we analyze the effective behavior for a set of samples created with different values of the starch and protein volume fractions and their interface adhesion. We compare our results with the existing data and conclude with the salient results of this work.

\section{Numerical model of wheat endosperm}

\subsection{Lattice element method}

The wheat endosperm can be described as a cohesive granular aggregate composed of starch granules inter-connected by a protein matrix (Fig. 1(a) and b). This typical microstructure is thus a multi-phase material with three bulk phases of starch, matrix and voids and their interfaces. For the simulations of this multi-phase representation of wheat endosperm, we used the Lattice Element Method (LEM), which has already been successfully used as a model for wheat endosperm fractionation in 2D (Topin et al., 2008, 2009a) and applied to investigate the fracture properties of cemented granular materials in 3D (Affes et al., 2012). This approach has also been extensively used in statistical physics of disordered media (Roux, 1990; Schlangen and Garboczi, 1996, 1997; Van Mier et al., 1997).

The LEM consists in discretizing all phases on a regular or irregular lattice. By allowing the mechanical information to be transmitted along a finite number of space directions, this representation allows for efficient simulation of a large number of different phases. Each node of the lattice belong to a phase and the mechanical properties such as elasticity, elasticity limit and plastic strains are carried by the links between nodes. When the two nodes of a link belong to the same phase, the link represents a 'bulk phase' whereas the links with nodes belonging to different phases carry the 'interface' properties between the phases. The mechanical behavior is thus fully implemented by 1 ) the distribution of nodes in space (or the type of lattice), 2) the mechanical information carried by a link.

The endosperm sample is composed of three bulk phases $\varphi$ : starch $s$, protein $p$ and void space $v$. We assume a quasi-brittle elastic behavior of starch and protein, as usually observed in mechanical tests for reasonably low moisture content (Haddad et al., 1999; Delwiche, 2000).

This implies that the links should be modeled as linear springs uniquely characterized by their stiffness $k^{\varphi}$ and breaking threshold $f_{c}^{\varphi}$. Hence, each link between nodes $i$ and $j$ transmits only a single radial force $f^{i j}$ related to the node displacements by

$f^{i j}=k^{i j}\left(\ell^{i j}-\ell_{0}\right)$

where $\ell_{0}$ is the equilibrium element length and $\ell^{i j}$ is the element length. When the radial force $f^{i j}$ reaches its breaking threshold $f_{c}$, the element is broken and its stiffness vanishes. As we shall see, this elemental linear behavior leads to a quasi-brittle behavior with linear elastic properties at the lattice scale with properties depending on the volume fractions of different phases (Fig. 1(c)). The starch-starch $(s s)$ and starch/protein $(s p)$ interfaces may have different values of stiffness and breaking threshold. The bulk links of the void phase and its interface with other phases have, by definition, zero stiffness.

In the following, we will express the element characteristics in stress units. Therefore, the yield stress $\sigma^{\varphi}$ of an element belonging to the phase $\varphi$ and its elastic modulus $E^{\varphi}$ are defined as 

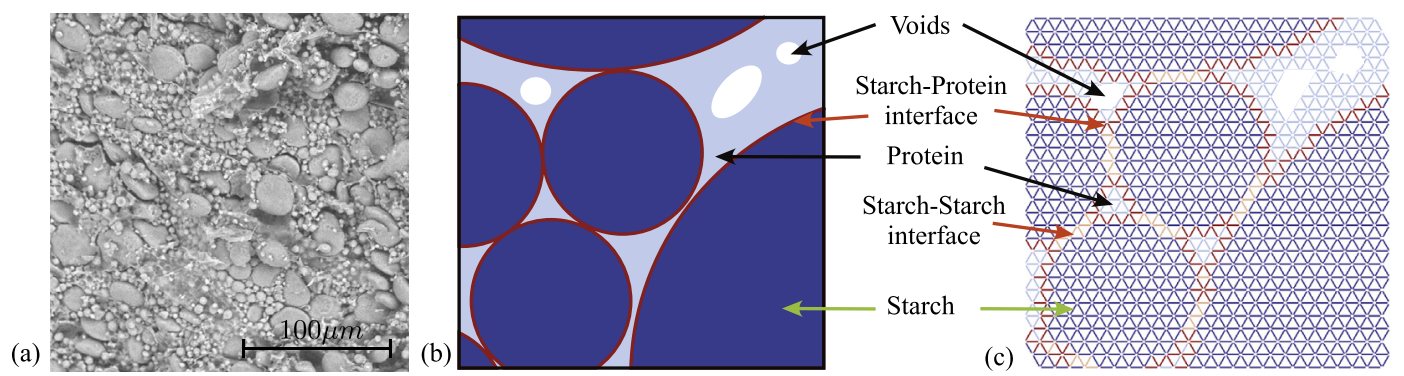

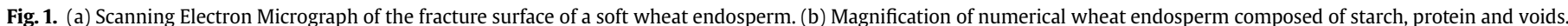
(c) Representation of the numerical wheat endosperm on a regular triangular lattice where starch-protein and starch-starch interfaces are highlighted.

$\sigma^{\varphi} \equiv f^{\varphi} / a$

and

$E^{\varphi} \equiv k^{\varphi} / a$

where $a$ is the free length of the lattice element. These elastic moduli $E^{\varphi}$ of the lattice are different from their equivalent phase moduli, which depends both on the bond moduli and the geometry of the lattice. Square brackets are thus used to represent the phase (both bulk and interface) moduli $E^{[s]}, E^{[p]}, E^{[s s]}$ and $E^{[s p]}$. In general, the phase modulus $E^{[\varphi]}$ is a linear function of the elemental modulus $E^{\varphi}$. For a triangular lattice, for example, we have (Schlangen and Garboczi, 1997):

$E^{[\varphi]}=\frac{\sqrt{3}}{2} E^{\varphi}$

In lattice representation, the overall interfaces $s s$ and $s p$ have nonzero volume fractions $\rho^{s s}$ and $\rho^{s p}$, respectively. These volumes are, however, small compared to the volume fractions $\rho^{s}, \rho^{p}$ and $\rho^{v}$ of bulk phases. For this reason, in the following we assume that they are part of the particle phase so that

$\rho^{S}+\rho^{p}+\rho^{v}=1$

Note that, although $s p$ and $s s$ are interfacial phases constituting a monolayer of elements between two phases, they affect the global behavior of the endosperm through their specific surface (total surface per volume unit).

Fig. 2 illustrates the numerical procedure used to solve the mechanical problem. Further details can be found in reference (Topin et al., 2007). The initial state is the reference configuration; see Fig. 2(1). The sample is then deformed by imposing desired displacements to the nodes belonging to the upper boundary as shown in Fig. 2(2). The total elastic energy of the system is a convex function of node displacements and thus finding the unique equilibrium configuration of the nodes implies a minimization problem. Performing this minimization for a stepwise loading corresponds to submitting the system to a quasi-static deformation process; Fig. 2(3). The overloaded elements (where calculated force are above their breaking thresholds) are removed. This corresponds to irreversible microcracking of the material at the element scale. The released elastic energy between two successive equilibrium states is fully dissipated by microcracking and a new equilibrium state is reached before the next strain increment is applied; Fig. 2(4).

\subsection{Sample generation and local properties}

We describe here the procedure used to generate the numerical samples and to assign local mechanical properties to the different phases and interfaces. Mature starchy endosperm of common wheat grain contains up to $80 \%$ of starch made of large (A-type, $d_{A}=20 \mu \mathrm{m}$ ) and small (B-type, $d_{B}=5 \mu \mathrm{m}$ ) granules (Goesaert et al., 2005; Massaux et al., 2008). For the numerical samples, the particles sizes are set randomly following a similar distribution as in Topin et al. (2008). with $d_{A}=4 \times d_{B}$ and $n_{B}=4 \times n_{A}$. The dense packings are composed of about 5000 bidisperse rigid disk-like particles; see Fig. 3(a). A Discrete Element Method (DEM) code (Delenne et al., 2008) was then used to pack the particles in a box at a controlled confining pressure. A classical linear spring-dashpot model for the contact law was used as well as a Coulomb law of friction with intergrains coefficient of friction $\mu=0.4$. This compaction process leads to a packing in mechanical equilibrium with a packing fraction depending on the confining pressure. Three different values of confining pressure were used to obtain three distinct DEM samples of starch volume fractions 0.73 (DEM1), 0.79 (DEM2) and 0.87 (DEM3), respectively. The DEM3 sample is shown in Fig. 3(a) with its boundary conditions.

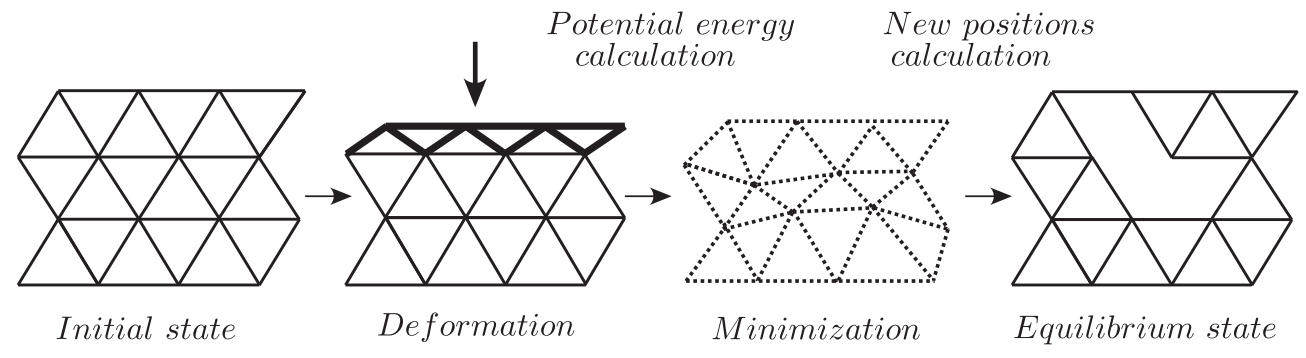

(1)

(2)

(4)

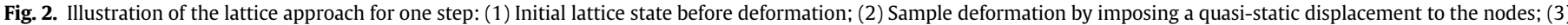

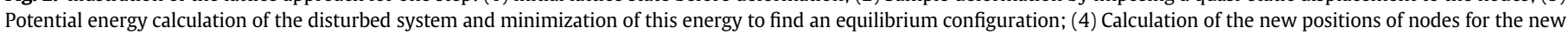
equilibrium state. 


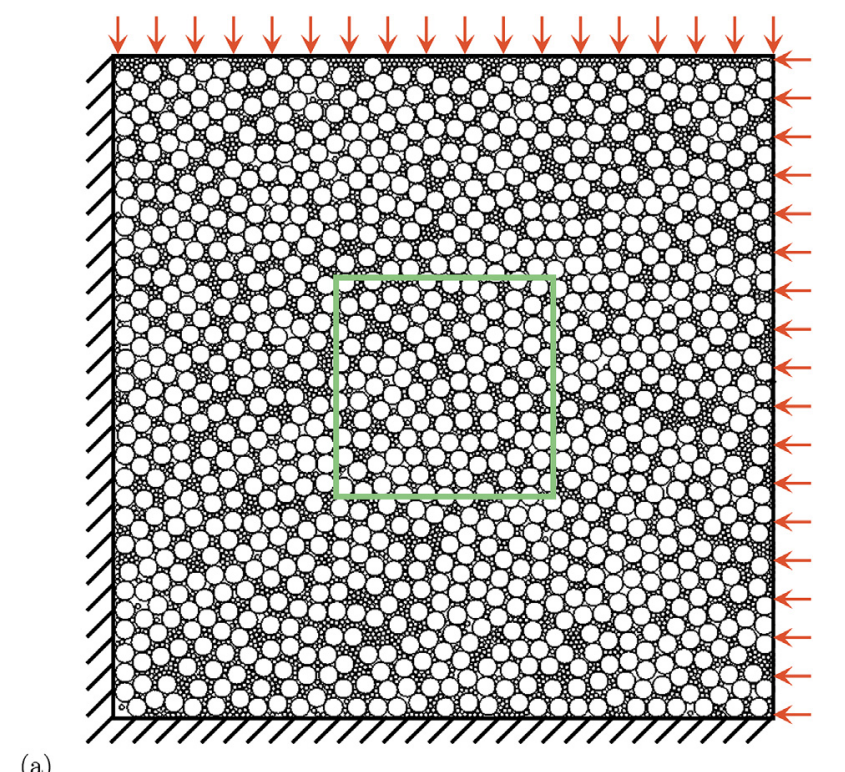

(a)

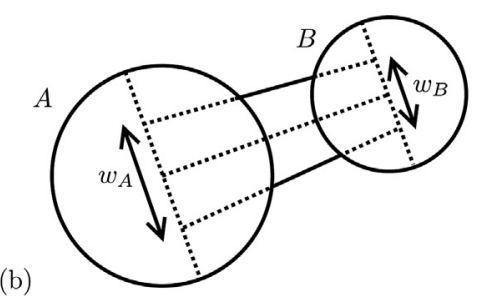

Fig. 3. (a) Bidisperse granular sample generated with DEM for the highest starch volume fraction (DEM3). The arrows indicate the isotropic compression imposed to upper and right boundaries. The final sample is represented by the green square after cropping. (b) Geometrical model of binding bridge between starch particles.

To avoid boundary effects in the LEM samples, we generate the latter from the DEM samples by cropping a square including 500 particles out of the central zone in the DEM sample. The cropped granular samples are then meshed with $M=2,20,000$ elements. This discretization provides an acceptable compromise between numerical efficiency, precision and representativity of the generated structures. Due to the finite size of mesh elements, the total volume fraction of discretized particle phase is 0.66 for DEM1, 0.72 for DEM2 and 0.80 for DEM3; see Table 1 . The DEM3 sample corresponds to largest volume fraction that could be reached for the particle phase (bidisperse random closed packing) and the DEM1 sample has the lowest particle volume fraction that could be obtained with a stable structure under load.

When the particle samples are generated, the protein phase is introduced in the form of trapezoidal-shaped bridges connecting neighboring particles. The bridges width $w$ is proportional to the radii of starch particles with $w=\alpha d$; Fig. 3(b). The protein volume fraction $\rho^{p}$ is varied from very low protein content to samples completely filled with proteins; Fig. 4(b). Note that $\rho^{p}$ includes the starch/protein $\left(\rho^{s p}\right)$ and starch/starch $\left(\rho^{s s}\right)$ interface volume fractions. The maximum value of $\rho^{p}$ depends on the particle volume

Table 1

Volume fraction of the starch particle $\left(\rho^{s}\right)$, protein $\left(\rho^{p}\right)$ and void $\left(\rho^{v}\right)$ phases according to the different levels of isotropic compression.

\begin{tabular}{llll}
\hline & DEM3 & DEM2 & DEM1 \\
\hline$\rho^{s}$ & 0.80 & 0.72 & 0.66 \\
$\rho^{p}$ & $0.03-0.20$ & $0.16-0.28$ & $0.16-0.34$ \\
$\rho^{v}$ & $0.17-0$ & $0.12-0$ & $0.18-0$ \\
\hline
\end{tabular}

fraction and zero porosity. In this limit, the sample is then totally vitreous; see Table 1. Higher porosities correspond to lower vitreousness or mealy endosperm.

Unlike (Topin et al., 2009a), who considered the same elastic moduli and breaking stresses for starch particles and protein matrix, the data of (Chichti et al., 2013) clearly shows differences in the nanomechanical properties of each polymers. Based on those results, the elastic moduli and breaking stresses for starch particles and protein matrix were set as $E^{S}=3.5 E^{p}$ with $E^{S p}=E^{S S}=E^{p}$ and $\sigma^{s}=2.5 \sigma^{p}$.

Furthermore (Topin et al., 2008, 2009a), also assumed zero adhesion at the starch-starch interface $\left(\sigma^{s s}=0\right)$, a condition that leads to 'bare' contacts between starch granules. However, recent microscopic analysis and AFM mechanical investigation, as described in (Chichti et al., 2013) (section 4.2) shows that in wheat endosperm the starch granules are generally coated by the protein matrix at the contact points between starch particles. Therefore, we set the $s s$ interface to have the same mechanical properties as the $s p$ interface. This choice will reduces finite mesh size effects for starch particles and removes the dependence of the contact area on the element size. The starch-protein adherence (the breaking stress $\sigma^{s p}$ ) was varied from $0.2 \sigma^{[s]}$, which corresponds to interface properties lower than those of the protein matrix, to $1.2 \sigma^{[s]}$. With this procedure, 90 fully meshed different LEM samples were obtained. Various parameters used to control the microstructure are summarized in Table 2.

All the above numerical samples were subjected to uniaxial tension tests until failure by imposing incremental displacement on the upper boundary. The lower boundary was fixed and the lateral boundaries were free to deform. We present and analyze below the stress transmission, stress-strain behavior and effective mechanical properties of the samples as a function of internal adhesion, starch particle volume fraction and protein volume fraction.

\section{Results and discussion}

\subsection{Stress transmission}

Fig. 4 shows three snapshots of samples with different packing fractions but with the same protein content $\left(\rho^{p}=0.16\right)$ and starchprotein adhesion $\left(\sigma^{s p}=1\right)$. Hence, only the void volume fraction changes. Although the protein content is the same, the samples represent thus porous (mealy), intermediate porous and nonporous (vitreous) materials. The vertical stress maps are shown before failure in 4(a). We observe tensile "stress chains" passing mainly through starch particles. Despite the contrasted stiffness of the protein matrix and starch particles, this figure clearly shows also that the pores concentrate stresses in the matrix bridges between the particles. The stress field is more inhomogeneous when the porosity is larger. The material is therefore more prone to failure in this case as a result of stronger stress concentration. In the case of the dense granular packing $\left(\rho^{s}=0.80\right)$, as the pore space is mainly filled by the protein matrix, the stress chains are distributed more evenly in the whole sample. As a result, the tensile strength and elastic moduli are expected to be higher in this limit.

The crack paths are shown in Fig. 4(b) for the same samples. In all cases, stress concentration in the vicinity of pores and contacts leads to initiation of cracks that propagate through the sample along the direction perpendicular to the direction of loading. The failure pattern appears, however, to be more diffuse for samples at high porosity and roughly follow a straight line in the case of low porosity. 

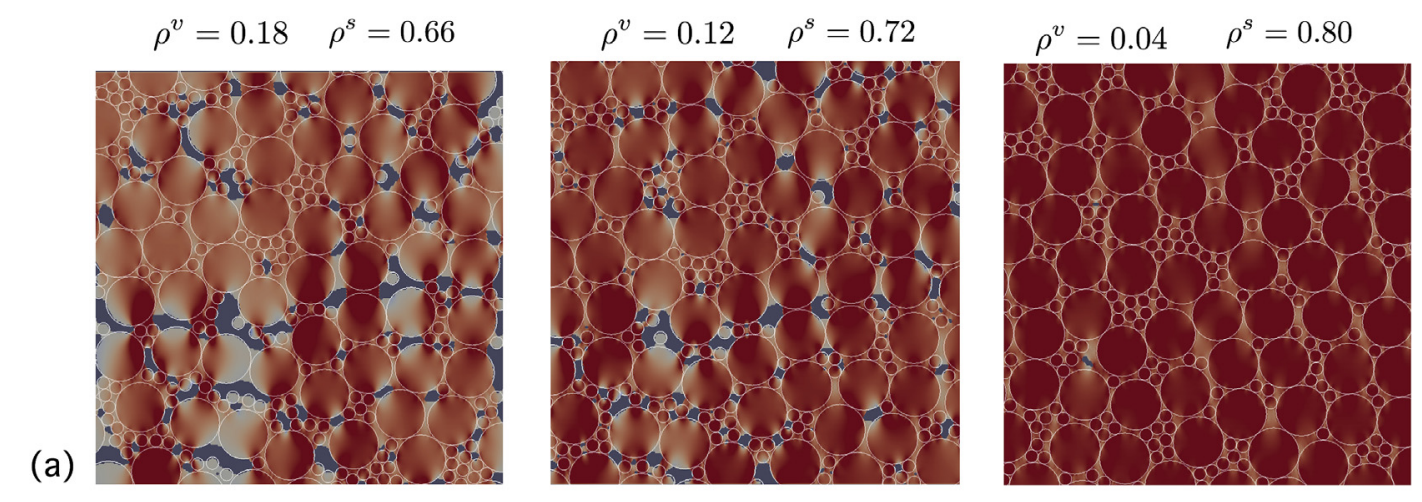

(b)
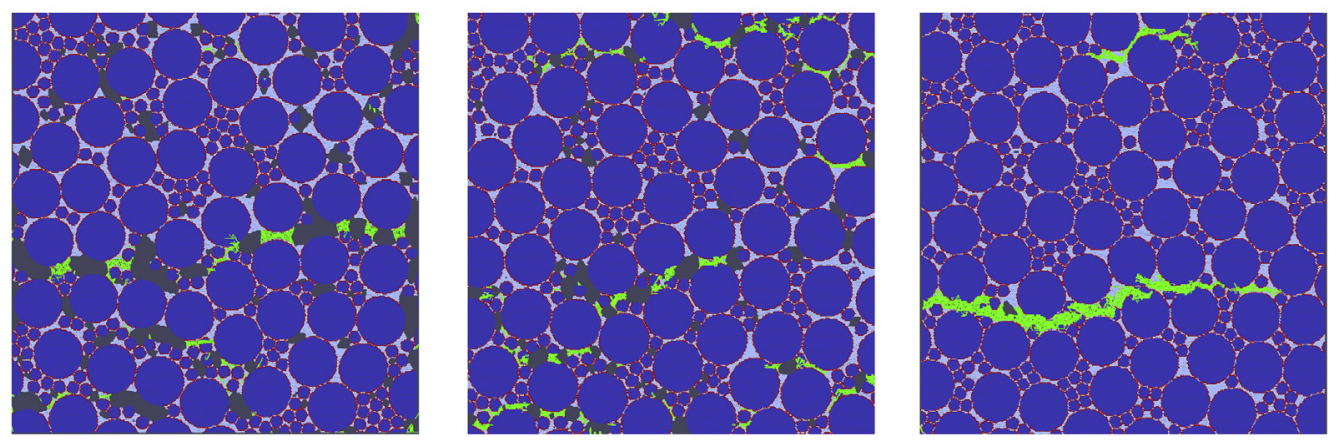

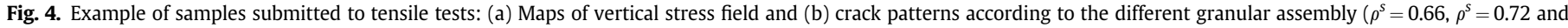
$\rho^{s}=0.80$ ) for $\rho^{p}=0.16$ and $\sigma^{s p}=1$. Maps correspond to focused zone taken out from the overall sample.

Table 2

Parameters controlling the microstructure and phases and interfaces mechanical properties.

\begin{tabular}{ll}
$\begin{array}{l}\text { Particle size distribution } \\
\text { Particle diameter } \\
\text { Number of particles }\end{array}$ & $\begin{array}{l}d_{A}=4 d_{B} \\
n_{B}=4 n_{A}\end{array}$ \\
& \\
Local mechanical properties & \\
Stiffness & $E^{s}=3.5 E^{p} ; E^{p}=E^{s p}$ \\
Tensile strength & $\begin{array}{l}\sigma^{s}=2.5 \sigma^{p} \\
\sigma^{s s}=\sigma^{s p} \\
\end{array}$ \\
Adhesion & $0.2 \sigma^{s}<\sigma^{s p}<1.2 \sigma^{s}$ \\
& \\
Phase distribution & \\
Matrix volume fraction & $0.10<\rho^{p}<0.34$ \\
Particle volume fraction & $0.66<\rho^{s}<0.80$ \\
\hline
\end{tabular}

\subsection{Stress-strain behavior}

Fig. 5 shows the relationship between tensile strain $\varepsilon$ and tensile stress $\sigma_{\text {eff }}$ for different values of the starch volume fraction $\rho^{s}$, starch-protein adhesion $\sigma^{s p}$ and void volume fraction $\sigma^{v}$. In all cases, we observe an elastic behavior with an effective stiffness $E_{\text {eff }}$ at low strain, followed by brittle failure at a well-defined value of the tensile stress $\sigma_{\text {eff. }}$ A similar brittle behavior has been observed in experiments performed on small cubic samples extracted from endosperm (Haddad et al., 1998, 1999, 2001). It is noteworthy that the plots of Fig. 5 seem to be less brittle than those obtained by Topin et al. (2009a). This discrepancy may be attributed to the fact that the cohesion between starch granules in our simulations is equal to that between starch granules and the protein whereas in previous simulations by Topin et al. it was set to zero.

The stress-strain plots of Fig. 5 highlight several features related to the sample porosity and starch-protein adhesion. The plots for extreme values of the starch-protein adhesion shown in Fig. 5(a) for $\sigma^{s p} / \sigma^{[s]}=0.2$ and $\sigma^{s p} / \sigma^{[s]}=1.2$ with $\rho^{v}=0.04$ correspond to soft and hard wheat classes. The volume fraction of starch particles has a stronger effect in samples displaying strong starch-protein adhesion. The dense granular packing with $\rho^{s}=0.80$ has the largest tensile strength compared to those with $\rho^{s}=0.72$ and $\rho^{s}=0.66$. At low starch-protein adhesion, no difference is observed among samples with different starch granule volume fractions.

Stress-strain behavior for $\rho^{v}=0$ and $\rho^{v}=0.10$ at the same starchprotein adhesion $\left(\sigma^{s p} / \sigma^{[s]}=1.2\right)$ are shown in Fig. 5 (b) as examples of non-porous (vitreous) and porous (mealy) kernel endosperms. The effect of starch volume fraction on tensile strength is significant especially at low values of $\rho^{v}$. For $\rho^{v}=0$, a dense granular packing results in high tensile strength (red solid lines in Fig. 5 (b)).

\subsection{Effective properties}

From the stress-strain data, we can estimate Young's modulus (in the elastic regime prior to stress peak) and tensile strength (peak stress) of the packings as a function of different parameters. Fig. 6(a) shows the evolution of effective Young's modulus $E_{\text {eff }}$ normalized by $E^{[s]}$ as a function of the protein matrix $\rho^{p}$ and starch volume fraction $\rho^{s}$, for all the values of $\sigma^{s p} / \sigma^{[s]}$. The previous data of Topin et al (Topin et al., 2008). are shown as well. $E_{\text {eff }}$ increases linearly with protein content $\rho^{p}$ for each given value of $\rho^{s}$ but its maximum value $\left(\simeq 0.75 E^{[s]}\right)$ is independent of $\rho^{s}$. Eeff is also independent of starch-protein adhesion $\sigma^{s p}$, which is only significant for the breaking threshold, except in the range of low adhesion where we observe early microcracking at contacts between particles even at low tensile stress. This leads to a small discrepancy in the measured modulus.

Fig. 6 (b) shows the evolution of $E_{\text {eff }}$ as a function of porosity $\rho^{v}$. We see that all our data points nearly collapse on the same curve, indicating that Young's modulus scales mainly with porosity irrespective of the relative proportions of protein and starch volumes fractions. The differences due to different values of the volume fractions or proper elastic stiffnesses of starch and protein exist but 


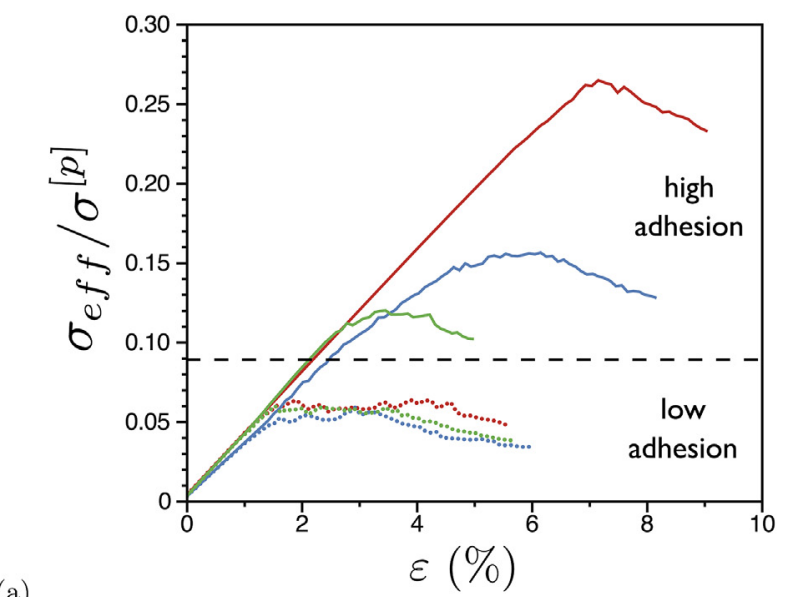

(a)

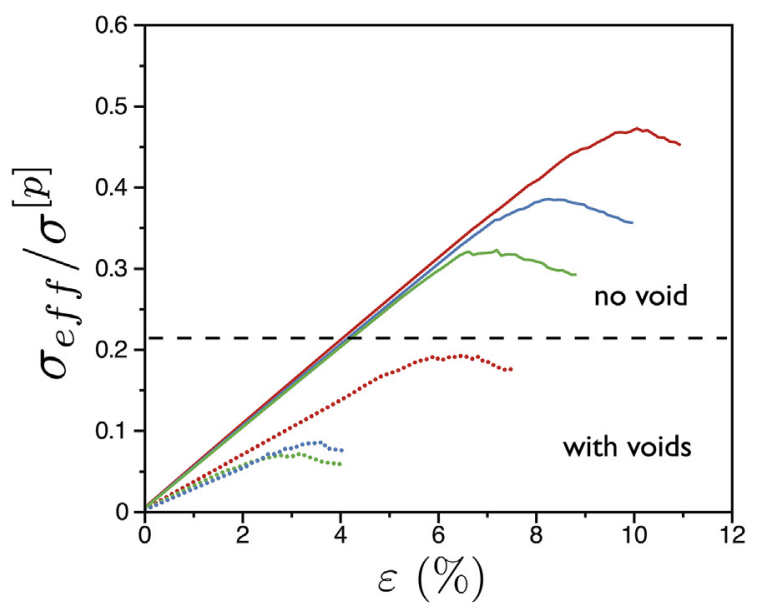

(b)

Fig. 5. Stress-strain plots showing the effect of different values of the starch particle volume fraction $\left(\rho^{s}=0.66\right.$ : green, $\rho^{s}=0.72$ : blue, $\rho^{s}=0.80$ : red) according to (a) starchprotein adhesion (solid line: $\sigma^{s p} / \sigma^{[s]}=1.2$, dashed lines $\sigma^{s p} / \sigma^{[s]}=0.2$ ) and (b) void volume fraction (solid line for $\rho^{v}=0$, dashed lines for $\rho^{v}=0.10$ ). (For interpretation of the references to colour in this figure legend, the reader is referred to the web version of this article.)

they are undermined by the effect of porosity. The variation is almost linear, a typical property of particle-reinforced composites, in which elastic properties depend on the presence of pores. There is, however, a major difference between wheat endosperm and composites: in composites the particle inclusions and pores are diluted and do not have direct mutual interactions. It is not the case in a cemented granular material such as wheat endosperm in which both the pores and the network of percolating particles lead to strong stress concentration. In this sense, the scaling of elastic behavior with porosity is a remarkable observation.

In order to characterize such effects arising from the presence of a granular backbone and pores, we consider the difference in effective elastic properties between our samples and composites made of the same proportions of protein, starch and voids, with voids and starch granules distributed in the form of cylindrical objects inside a proteic matrix. The theoretical effective Young's modulus $E^{\text {th }}$ can be determined from the analytical solution of Mori and Tanaka (1973). For our system, this solution is given by the following relation:

$E_{e f f}^{t h}=\frac{d^{p} E^{[p]}+d^{s} E^{[s]}}{e^{p} E^{[p]}+e^{s} E^{[s]}} E^{[p]}$

where

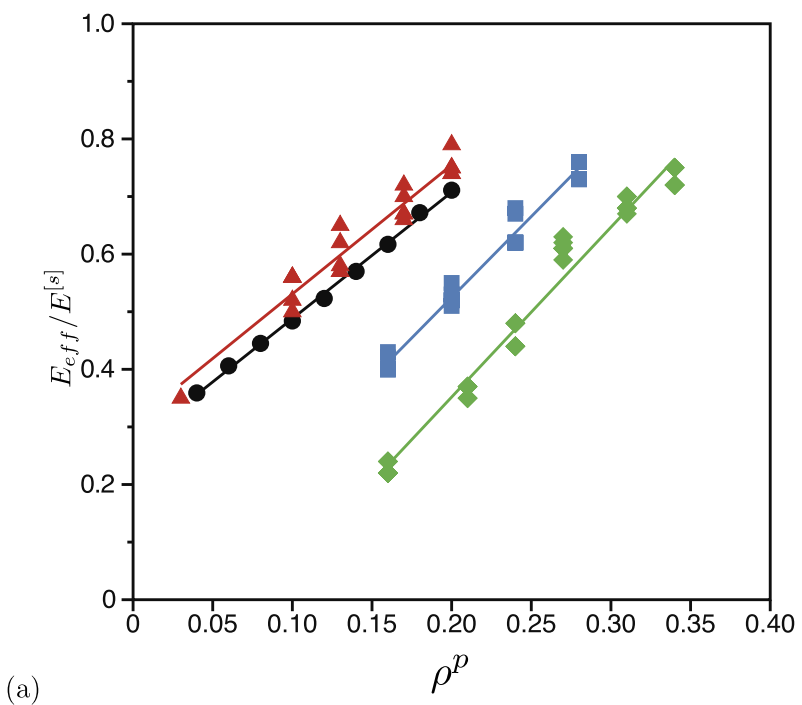

(a)

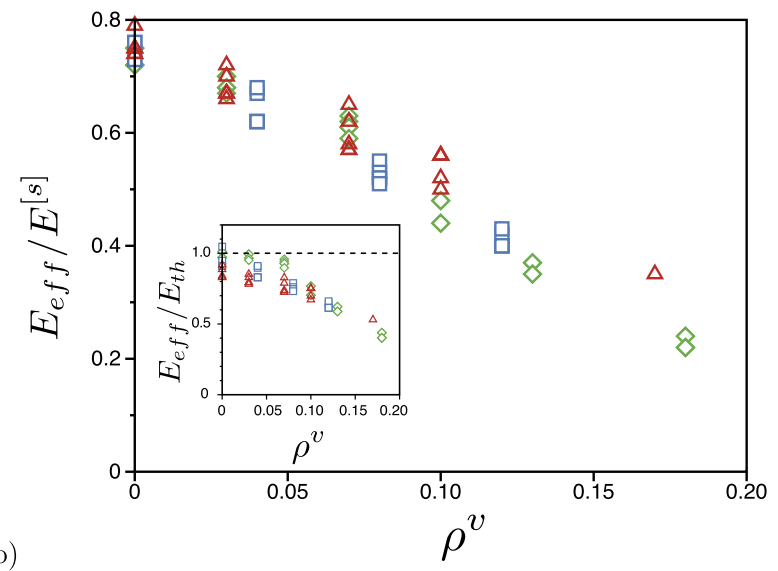

Fig. 6. Effective Young's modulus as a function of (a) protein volume fraction $\rho^{p}$ and (b) porosity $\rho^{v}$ for different values of the starch particle volume fraction: $\rho^{s}=0.80$ (red triangles), $\rho^{s}=0.72$ (blue squares) and $\rho^{s}=0.66$ (green diamonds). Black circles are those obtained in (Topin et al., 2008) for tensile loading. The solid lines are linear fits to the data points. The inset shows the ratio of measured modulus as a function of $\rho^{v}$ using Mori-Tanaka analytic expression. (For interpretation of the references to colour in this figure legend, the reader is referred to the web version of this article.)

$d^{p}=16 \rho^{p} A^{2}$

$d^{S}=12 A \rho^{p}+(3+4 A) \rho^{S}$

$e^{p}=12 A\left(1-\rho^{p}\right)+16 A^{2}$

$e^{s}=12 A\left(1-\rho^{s}\right)+9 \rho^{v}$

and in which $A=2(1-\nu) /(1+\nu)$ in $2 D$ and represents the ratio of the shear modulus to the bulk modulus of a material of Poisson's ratio $\nu$ ( $\nu \simeq 0.3$ in this work). It is important to note that this model is only valid for a composite material involving two populations of diluted spherical inclusions (starch particles and voids) inside a matrix and makes no distinction between tension and compression (Hu et al., 1998).

The ratios of the measured Young's modulus to the Mori-Tanaka prediction (which does not account for contact and interfaces) for all our samples are plotted in the inset of Fig. 6(b). We see that the prediction is fairly good at low porosity (nearly continuous material) but it gets increasingly less accurate at high $\rho^{v}$ values when the 
underlying granular microstructure is revealed due to the presence of pores. Consequently this shows that the wheat endosperm does not behave as a simple composite in which the particles are diluted into a matrix. This result is in agreement with the data of (Topin et al., 2007) and consistent with the fact that the probability density function of stresses in the system is increasingly broader for decreasing matrix content as formerly described in (Topin et al., 2009b). For a low matrix volume fraction (and thus at high porosity), high stress concentrations are located at the binding bridges between the particles and this leads to a strong discrepancy with the composite model. In our case, this effect is enhanced by the difference in stiffness between starch and matrix, which is an additional source of heterogeneity. Higher discrepancy occurs in the case of shearing or compressive tests as shown by (Topin et al., 2007).

Fig. 7 shows grey level maps of the tensile strength as a function of $\rho^{v}$ and starch-protein adhesion $\left(\sigma^{s p} / \sigma^{[s]}\right)$ for three values of starch volume fraction. The maps exhibit a non-linear evolution of the tensile strength with respect to both parameters. These maps clearly indicate that the strength increases with starch-protein adhesion $\sigma^{s p}$ but also with starch volume fraction $\rho^{s}$. As expected, it decreases as porosity increases. A small increase in porosity leads to a rapid falloff of the tensile strength so that the difference of

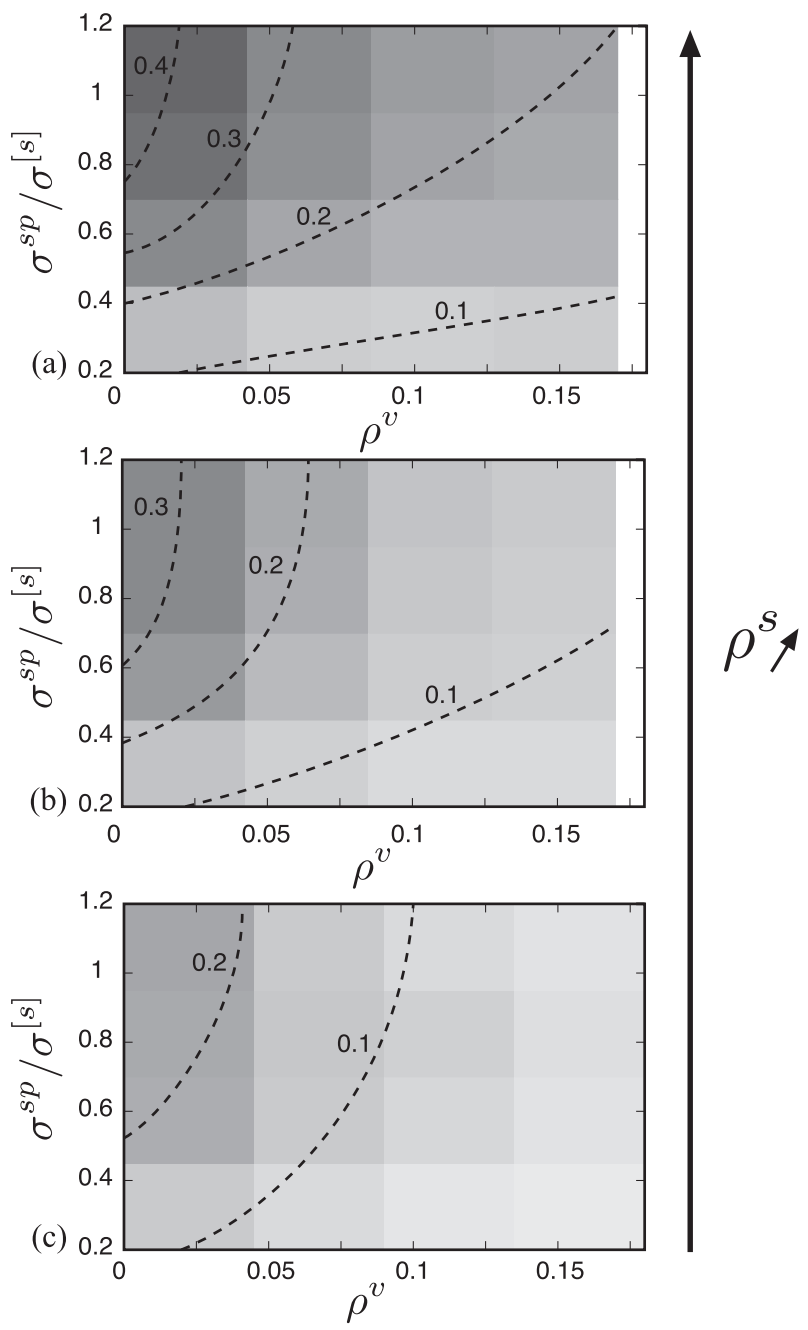

Fig. 7. Grey level map of tensile strength in the parameter space of starch-protein adhesion $\left(\sigma^{s p} / \sigma^{[s]}\right)$ and void volume fraction $\left(\rho^{v}\right)$ for the three different values of starch volume fraction: $\rho^{s}=0.80(\mathrm{a}), \rho^{s}=0.72$ (b) and $\rho^{s}=0.66$ (c). strength between high and low values of starch-protein adhesion (corresponding respectively to hard-like and soft-like wheats) is more important at low $\rho^{v}$ (vitreous state) compared to high $\rho^{v}$ (mealy state). This observation is consistent with experiment measurements of the mechanical properties of wheat endosperm under compression (Haddad et al., 1998, 1999, 2001).

\section{Conclusions}

In this paper, the wheat endosperm is modeled as a cemented granular aggregate composed of starch granules and a binding protein matrix. This model provides a framework in which the mechanical properties of wheat endosperm can be simulated as a function of starch-scale properties such as starch-protein adhesion and micro-structural parameters (starch granules volume fraction and protein volume fraction), and mapped onto textural properties of wheat endosperm such as hardness and vitreousness.

The bottom-up approach employed in this work is based on the Lattice Element Method (LEM), which allows us to simulate the mechanical behavior and fracture of numerical samples with variety of textures and local properties. This method uses a lattice-type sub-particle discretization of both starch granules and protein matrix. It allows for crack initiation and propagation by debonding of lattice elements. An important new ingredient of the present work is that the mechanical parameters were directly determined at the scale of starch granules and protein matrix by means of AFM measurements (Chichti et al., 2013).

The simulations showed that the porosity outweighs the effect of other parameters for the elastic modulus but are affected also by the granular backbone composed of percolating starch granules. Stress concentration depends both on porosity and the network of contacts between starch granules. As a result, a strong discrepancy was observed between the numerically measured effective Young's modulus and the mean-field approach based on the assumption of a homogeneous distribution of phases in space. This indicates that the wheat endosperm should not be considered as a simple particle-enriched composite.

Another important point that was highlighted for the first time in this investigation is the crucial role of starch granule volume fraction and connectivity for the tensile strength of wheat endosperm. This is the main reason why the protein content cannot be fully correlated to vitreousness, and samples of similar protein content can be different in vitreosity. Since the effect of starch granule volume fraction has not yet been studied in real experiments, our results suggest that it can be a key feature to be investigated in the future for understanding the strength variability. It was also shown that the interface adhesion has strong impact on the tensile strength, and that the volume fraction of starch granules affects the strength mainly at high interface adhesion.

This work may be pursued along several research directions. In particular, the packing effects may be further analyzed by considering the role of starch granule size distribution and the shape of large granules which are frequently elongated. Regarding the starch-scale properties, an interesting issue is the effect of local ductile breakage on the global tensile strength. In the present work, the local behavior was implemented by using brittle 1D elements. However, in the presence of water in the grinding process, the fracture of wheat grains is ductile. Such a behavior can in principle be introduced in a LEM approach through a damage parameter at the elemental scale. Another interesting aspect is the effect of dynamic loading on the strength and failure of endosperm. Starch damage, which is an important parameter for end-use quality of flours, can be studied for different loading rates representing the milling power. 


\section{References}

Affes, R., Delenne, J.-Y., Monerie, Y., Radjai, F., Topin, V., 2012. Tensile strength and fracture of cemented granular aggregates, Eur. Phys. J. E 35, 1-15.

Anjum, F., Walker, C., 1991. Review on the significance of strach and protein to what kernel hardness. J. Sci. Food Agric. 56, 1-13.

Barlow, K.K., Buttrose, M.S., Simmonds, D.H., Vesk, M., 1973. The nature of the starch-protein interface in wheat endosperm. Cereal Chem. 50, 443-454.

Beecher, B., Bettge, A., Smidansky, E., Giroux, M., 2002. Expression of wild-type pinb sequence in transgenic wheat complements a hard phenotype. Theor. Appl. Genet. 105, 870-877.

Chichti, E., George, M., Delenne, J.-Y., Radjai, F., Lullien-Pellerin, V., 2013. Nanomechanical properties of starch and gluten biopolymers from atomic force microscopy. Eur. Polym. J. 49 (12), 3788-3795.

Cundall, P. Strack, O., 1979. A discrete numerical model for granular assemblies. Geotechnique 29, 47-65.

Dai, Z., Yin, Y., Wang, Z., 2009. Starch granule size distribution from seven wheat cultivars under different water regimes. Cereal Chem. 86, 82-87.

Delenne, J., Haddad, Y., Benet, J., Abecassis, J., 2008. Mechanics of cohesive granular media for analysis of hardness and vitreousness of wheat endosperm. J. Cereal Sci. 47, 438444.

Delwiche, S., 2000. Wheat endosperm compressive strength properties as affected by moisture. Trans. ASAE 43, 365-373.

Dobraszczyk, B., Whitworth, M., Vincent, J., Khan, A., 2002. Single kernel wheat hardness and fracture properties in relation to density and the modelling of fracture in wheat endosperm. J. Cereal Sci. 35, 245-263.

Evers, T., Millar, S., 2002. Cereal grain structure and development: some implications for quality. J. Cereal Sci. 36, 261-284.

Giroux, M., Morris, C., 1998. Wheat grain hardness results from highly conserved mutations in the friabilin components puroindoline a and b. Proc. Natl. Acad. Sci. U. S. A. 95, 6262-6266.

Glenn, G., Johnston, R., 1992. Mechanical properties of starch, protein and endosperm and their relationship to hardness in wheat. Food Struct. 11, 187-199.

Goesaert, H., Brijs, K., Veraverbeke, W., Courtin, C., Gebruers, K., Delcour, J., 2005 Wheat flour constituents: how they impact bread quality, and how to impact their functionality. Trends Food Sci. Techol. 16, 12-30.

Greenwell, P., Schofield, J., 1986. A starch granule protein associated with endosperm softness in wheat. Cereal Chem. 63, 379-380.

Greffeuille, V., Abecassis, J., Rousset, M., Oury, F.-X., Faye, A., Bar L'Helgouac'h, C., Lullien-Pellerin, V., 2006. Grain characterization and milling behaviour of nearisogenic lines differing by hardness. Theor. Appl. Genet. 114, 1-12.

Greffeuille, V., Abecassis, J., Barouh Villeneuve, N., Mabille, F., Bar L'Helgouac'h, C., Lullien-Pellerin, V., 2007. Analysis of the milling reduction of bread wheat farina: physical and biochemical characterisation. J. Cereal Sci. 45, 97-105.

Haddad, Y., Benet, J., Abecassis, J., 1998. A rapid general method for appraising the rheological properties of the starchy endosperm of cereal grains. Cereal Chem. 75, 673-676.

Haddad, Y., Mabille, F., Mermet, A., Abecassis, J., Benet, J., 1999. Rheological properties of wheat endosperm with a view on grinding behaviour. Powder Tech. 105, 89-94.

Haddad, Y., Benet, J., Delenne, J., Mermet, A., Abecassis, J., 2001. Rheological behaviour of wheat endosperm-proposal for classification based on the rheological characteristics of endosperm test samples. J. Cereal Sci. 34, 105-113.

Hogg, A., Sripo, T., Beecher, B., Martin, J., Giroux, M., 2004. Wheat puroindolines interact to form friabilin and control grain hardness. Theor. Appl. Genet. 108 1089-1097.

Hu, G., Guo, G., Baptiste, D., 1998. A micromechanical model of influence of particle fracture and particle cluster on mechanical properties of metal matrix composites. Comp. Mater. Sci. 9, 420430.
Hurkman, W., Wood, D., 2011. High temperature during grain filling alters the morphology of protein and starch deposits in the starchy endosperm cells of developing wheat (Triticum aestivum L.) grain. J. Agric.Agric. Food Chem. 59, 4938-4946

Lopez-Ahumada, G.A., Ramirez-Wong, B., Torres-Chavez, P.I., Bello-Perez, L., Figueroa-Cardenas, J.D., Garzon-Tiznado, J., Gomez-Aldapa, C., 2010. Physicochemical characteristics of starch from bread wheat (triticum aestivum) with "yellow berry". Starch 62, 517-523.

Massaux, C., Sindic, M., Lenartz, J., Sinnaeve, G., Bodson, B., Falisse, A., Dardenne, P. Deroanne, C., 2008. Variations in physicochemical and functional properties of starches extracted from euopean soft wheat (triticum aestivum 1.): the importance to preserve the varietal identity. Carbohyd. Polym. 71, 32-41.

Mori, T., Tanaka, K., 1973. Average stress in matrix and average elastic energy of material with misfitting inclusions. Acta Metall. 21, 571-574.

Morris, C., 2002. Puroindolines: the molecular genetic basis of wheat grain hardness. Plant Mol. Biol. 48, 633-647.

Ni, Y., Wan, Z., Yin, Y., Li, W., Yan, S., Cai, T., 2012. Starch granule size distribution in wheat grain in relation to phosphorus fertilization. J. Agric.Agric. Sci. 150, $45-52$.

Oury, F.-X., Lasme, P., Michelet, C. Rousset, M., Abecassis, J., Lullien-Pellerin, V., 2015. Relationships between wheat grain physical characteristics studied through near-isogenic lines with distinct puroindoline-b allele. Theor. Appl. Genet. 128, 913-929.

Parish, J., Halse, N., 1969. Effects of light, temeperature, and rate of dessication on translucency in wheat grain. Aust. J. Agric. Res. 19, 365-372.

Pasha, I., Anjum, A., Morris, C., 2010. Grain hardness: a major determinant of wheat quality. Food Sci. Tech. Int. 16, 511-522.

Roux, S., 1990. Statistical Models for Fracture in Disordered Media. North Holland, Amsterdam, pp. 87-114. Ch. Continuum and discrete description of elasticity and other rheological behavior.

Schlangen, E., Garboczi, E.J., 1996. New method for simulating fracture using an elastically uniform random geometry lattice. Int. J. Eng. Sci. 34 (10), 1131-1144.

Schlangen, E., Garboczi, E.J., 1997. Fracture simulations of concrete using lattice models: computational aspects. Eng. Fract. Mech. 57 (2-3), 319-332.

Topin, V., Delenne, J.-Y., Radjai, F., Brendel, L., Mabille, F., 2007. Strength and failure of cemented granular matter, Eur. Phys. J. E 23, 413-429.

Topin, V., Radjai, F., Delenne, J.-Y., Sadoudi, A., Mabille, F., 2008. Wheat endosperm as a cohesive granular material. J. Cereal Sci. 47, 347-356.

Topin, V., Radjai, F., Delenne, J.-Y., Mabille, F., 2009. Mechanical modelling of wheat hardness and fragmentation. Powder Tech. 109, 215-220.

Topin, V., Radjai, F., Delenne, J.-Y., 2009. Subparticle stress fields in granular solids. Phys. Rev. E 79, 051302

Turnbull, K.-M., Rahman, S., 2002. Endosperm texture in wheat. J. Cereal Sci. 36, 327-337.

Van Mier, J.G.M., Chiaia, B.M., Vervuurt, A., 1997. Numerical simulation of chaotic and self-organizing damage in brittle disordered materials. Comput. Methods Appl. Mech. Eng. 142 (1-2), 189-201.

Wang, L., Jeronimidis, G., 2008. Investigation of the fracture mode for hard and soft wheat endosperm using loading-unloading bending test. J. Cereal Sci. 48, 193-202.

Weightmann, R., Millar, S., Alava, J., Foulkes, M., Fish, L., Snape, J., 2008. Effects of drought and the presence of the $1 \mathrm{bl} / 1 \mathrm{rs}$ translocation on grain vitreosity, hardness and protein content in winter wheat. J. Cereal Sci. 47, 457-468.

Williams, P., Sobering, D., 1986. Attempts at standardization of hardness testing of wheat 1 the grinding sieving (particle size index) method. Cereal Foods World 31, 359-364.

Zhang, T., Wang, Z., Cai, Y.Y.Y., Yan, S., Li, W., 2010. Starch content and granule size distribution in grains of wheat in relation to post-anthesis water deficits. J. Agron. Crop. Sci. 196, 1-8. 